\title{
Conflictos por el agua en las cuencas de los ríos Matanza-Riachuelo y Reconquista. Claves para pensar la justicia hídrica a escala metropolitana
}

Gabriela Merlinsky

Universidad de Buenos Aires. Facultad de Ciencias Sociales. Instituto de Investigaciones Gino Germani. Buenos Aires, Argentina.

CONICET. Buenos Aires, Argentina.

\section{Melina Tobías}

Universidad de Buenos Aires. Facultad de Ciencias Sociales. Instituto de Investigaciones Gino Germani. Buenos Aires, Argentina.

CONICET. Buenos Aires, Argentina.

Recibido: 08 de junio de 2021. Aceptado: 29 de julio de 2021.

\section{Resumen}

A través del presente artículo proponemos reflexionar en torno a la justicia hídrica en la metrópolis de Buenos Aires. Para ello hemos desarrollado un análisis de los principales vectores de la desigualdad socio espacial en materia de agua, saneamiento y riesgo de inundaciones a escala metropolitana, concentrándonos en dos territorios hidrosociales: las cuencas de los ríos Matanza-Riachuelo y la del río Reconquista. Con relación a dichos territorios, interesa problematizar la construcción de nuevos significados en torno a la justicia hídrica y al modo en que estos disputan las concepciones prevalecientes acerca de la gestión de cuencas en diferentes escalas. Para cumplir estos objetivos, la metodología adoptada se basó en la triangulación de fuentes primarias (entrevistas en profundidad a miembros de organizaciones territoriales de las cuencas analizadas) y de fuentes secundarias (estadísticas oficiales de cobertura de servicios de agua y saneamiento, indicadores ambientales de inundación, entre otros). Entre las principales conclusiones, el trabajo permitió entrever que, si bien en los últimos años han tomado relevancia las cuencas como unidades de gestión y política ambiental, estas iniciativas no logran abordar el agua desde un enfoque integral que resguarde el derecho a la salud y a un hábitat digno. 


\title{
Water conflicts in the Matanza-Riachuelo and Reconquista river basins. Keys to think water justice at the metropolitan scale
}

\begin{abstract}
Through this article we propose to think about water justice in the metropolis of Buenos Aires. To this end, we have analyzed the main vectors of socio-spatial inequality in terms of water, sanitation and flood risk on a metropolitan scale, focused on two hydrosocial territories: the Matanza-Riachuelo river and the Reconquista river basins. Regarding these territories, we are interested in problematizing the development of new meanings around water justice and the way in which they dispute the prevailing conceptions about the management of basins at different scales. To achieve these objectives, the adopted methodology was based on the triangulation of primary sources (in-depth interviews with members of territorial organizations of the mentioned basins) and secondary sources (official statistics on coverage of water and sanitation services, flood environmental indicators, etc.). Among the main conclusions, the work allowed us to realize that, although in recent years basins have become relevant as units of management and environmental policy, these initiatives fail to address water from a comprehensive approach that protects the right to health and a decent habitat.
\end{abstract}

KEYWORDS: WATER JUSTICE. CONFLICTS. INEQUALITIES. METROPOLITAN BASINS.

PALAVRAS-CHAVE: JUSTIÇA HÍDRICA. CONFLITOS. DESIGUALDADES. BACIAS

METROPOLITANAS.

\section{Introducción}

En diciembre de 2020 el agua comenzó a cotizar en el mercado de futuros de Wall Street, algo que pone en evidencia su centralidad para las condiciones de reproducción del capital. Sin embargo, el agua no debe ser considerada una mercancía, muy por el contrario, es un bien común mal distribuido. En la Argentina, cerca de diez millones de personas no tienen acceso a una red pública que los abastezca y cerca de veinte millones no tienen cobertura de saneamiento (INDEC, 2010), circunstancias que son centrales para el acceso a un hábitat y condiciones de vida dignas.

Los problemas de falta de inversión y discontinuidad en la provisión de infraestructuras, el desigual reparto del agua entre provincias, el rol dominante de industrias, productores agrícolas y mega emprendimientos mineros en el manejo del agua -con el desvío de cauces, el uso de grandes volúmenes de agua en procesos extractivos y la contaminación de cursos por el uso creciente de pesticidas- están en la base de un reparto desigual de este bien común, lo que produce serias consecuencias para la salud y el ambiente.

Estas situaciones de injusticia hídrica (Boelens et al., 2011) adquieren ribetes particulares en las grandes ciudades de América Latina, pues su integración en regiones económicas globales está apalancada por un urbanismo flexible, cuyo principal objetivo es territorializar inversiones de gran escala en el entorno construido y así canalizar flujos de capital transnacional. Esto tiene consecuencias muy importantes sobre el uso del suelo, así como en la generación y distribución de las rentas que surgen de los procesos de urbanización. 
Todo ello implica una reconfiguración muy profunda de la cuestión urbana, algo que tiene enorme gravitación en los conflictos por el agua. Dado que el suelo es un bien económico sujeto a especulación, son los sectores populares los más afectados por este proceso de comodificación del suelo urbano, siendo desplazados, en muchos casos, a vivir en asentamientos informales. Territorios que, en general, se caracterizan por elevados niveles de degradación ambiental en los que se combinan situaciones de pobreza, altas tasas de crecimiento poblacional, riesgos de inundaciones, contaminación del suelo y el aire, e infraestructuras de agua y saneamiento de cobertura incompleta o calidad precaria.

De este modo, analizar la producción, circulación y distribución del agua en la ciudad representa una entrada más para entender el modo en que se disponen las desigualdades espaciales urbanas, pues no se trata apenas de cuestiones técnicas asociadas a la escasez o abundancia de la misma, por el contrario, la cuestión más importante son las decisiones políticas que inciden en este proceso (Swyngedouw, 2004). La circulación del agua se conecta con los flujos de poder que sostienen privilegios en áreas y sectores sociales que están plenamente integrados a partir de los bienes y servicios de la metrópolis, y ello a costa de la exclusión y marginación de otras zonas y grupos que deben autoproducir de forma precaria estos mismos bienes y servicios.

En el área metropolitana de Buenos Aires, y desde comienzos de este milenio, diversos grupos de afectados por problemas de acceso al agua y por la exposición a inundaciones recurrentes han desarrollado diferentes estrategias de acción colectiva para lograr la accesibilidad al recurso y también para ejercer influencia sobre modalidades de gestión del riesgo. Esto implica un proceso de valoración social que pone en evidencia que los flujos del agua son manipulados por determinadas geografías del poder, algo que implica la constitución de territorios hidrosociales (Boelens et al., 2016; Swyngedouw, 2009).

La concepción de territorio hidrosocial integra el concepto físico de cuenca con la dinámica social que se desarrolla en esta área; es decir, con distintos modos de concebir un recurso hídrico, sus diferentes usos sociales y su conexión con los flujos del agua y la infraestructura hidráulica (Orlove y Caton, 2010; Swyngedouw y Williams, 2016). Estos territorios se relacionan con el ciclo hidrosocial del agua, es decir con el funcionamiento de las cuencas. Sin embargo, mientras estas últimas se establecen a partir de contornos físicos, las fronteras de los territorios hidrosociales son constantemente recreadas y reconfiguradas por los actores sociales, pudiendo incluso sobrepasar o segmentar las demarcaciones de las cuencas (Damonte y Lynch, 2016). Por esa razón es muy importante estudiar la relación entre los flujos del agua urbana (relativamente naturales, intensamente construidos) y las cuencas hídricas.

Las cuestiones del agua son centrales en diferentes conflictos ambientales urbanos pues expresan la lucha por el derecho a la ciudad. Cuando hacemos referencia a la justicia hídrica, retomamos una conceptualización de "justicia" o "equidad" que asume su carácter relacional y que reconoce tanto las dimensiones materiales y económicas como los aspectos culturales. Esto abarca disputas sobre los recursos, pero también sobre el ejercicio de la autoridad, las reglas y derechos y, en términos más generales, las formas discursivas que justifican un reparto desigual del agua (Boelens y Zwarteveen, 2005). 
Este artículo plantea una reflexión en torno a la justicia hídrica en la metrópolis de Buenos Aires. Nuestro enfoque pretende mostrar de qué manera se retroalimentan, por un lado, la falta de acceso y/o la inequidad en el acceso al servicio de agua y saneamiento y, por el otro, la exposición a las inundaciones periódicas para producir un patrón de desigualdades. Para ello desarrollamos un análisis en dos pasos. Primero, presentamos los principales vectores de la desigualdad socioespacial en materia de agua, saneamiento y riesgo de inundaciones a escala metropolitana. Luego nos concentramos en dos territorios hidrosociales: la cuenca de los ríos Matanza-Riachuelo (CMR) y la cuenca del río Reconquista (CRR). Aquí nos interesa problematizar la construcción de nuevos significados en torno a la justicia hídrica y al modo en que estos sentidos disputan las concepciones prevalecientes acerca de la gestión de cuencas en diferentes escalas. Como plantean Paerregaard y Andersen: "la gobernanza del agua sigue siendo un campo disputado que no siempre sigue las reglas de los que están en el poder, sino que deja espacio para otros agentes, tanto humanos como no humanos, incluida el agua misma" (2019:464).

\section{Las desigualdades hídricas en Buenos Aires}

La Región Metropolitana de Buenos Aires (RMBA) es el territorio más poblado de la Argentina con más de quince millones de habitantes, distribuidos en áreas urbanas, peri-urbanas y rurales. La gran mayoría de estos habitantes (más de trece millones) radica en el Área Metropolitana de Buenos Aires (AMBA) conformada por la Ciudad Autónoma de Buenos Aires (CABA) y los veinticuatro partidos colindantes del Gran Buenos Aires (GBA). Como toda metrópolis de América Latina, el AMBA fue aumentando su tamaño a lo largo del tiempo de manera heterogénea, dando lugar al incremento de desigualdades socio-territoriales y ambientales. Mientras el eje norte se consolidó como la región más favorecida en términos de recursos económicos, servicios e infraestructura urbana; la zona sur -históricamente asociada al desarrollo industrial del país- presenta altos niveles de Necesidades Básicas Insatisfechas (NBI), falta de servicios públicos y degradación ambiental.

El AMBA posee diversas fuentes de aguas subterráneas y superficiales. Entre ellas, el Río de la Plata, perteneciente a la cuenca del Plata -principal fuente de agua dulce de la región-, y múltiples cuencas, arroyos y acuíferos que la atraviesan como son las cuencas del Río Luján, Matanza-Riachuelo y Reconquista; los arroyos entubados que recorren la CABA como el Maldonado, Cildáñez, Vega, Medrano, entre otros, y los acuíferos Pampeano y Puelche utilizados para extracción y abastecimiento (Solo et al., 1990:14). Esta característica define una peculiaridad pues, a diferencia de otras regiones de la Argentina en donde el recurso hídrico es escaso, en esta región el agua se encuentra presente "en todas partes". 


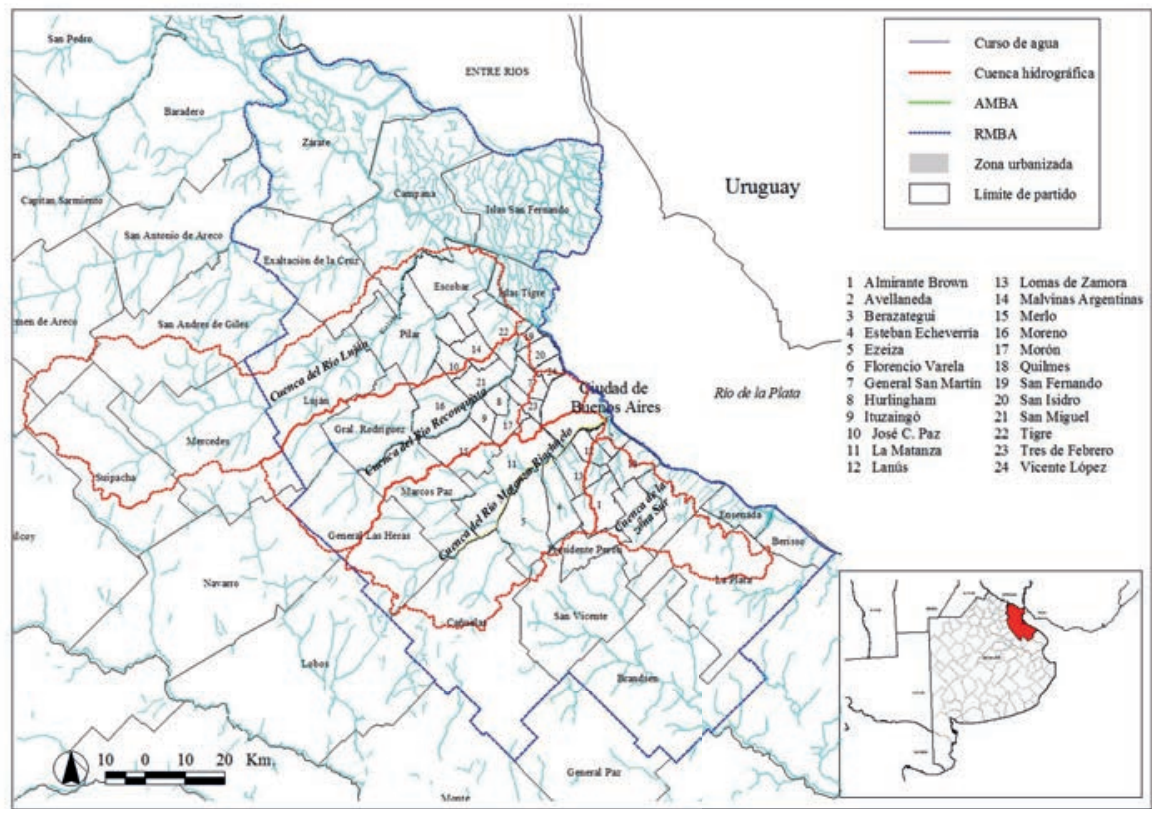

Figura 1. Cuencas metropolitanas de Buenos Aires. Fuente: Garay y Fernández (2013).

No obstante, a pesar de contar con una multiplicidad de fuentes, estas no necesariamente garantizan el acceso universal ni equitativo al recurso ni al servicio. Esto puede observarse al analizar la accesibilidad a los servicios de agua y saneamiento: en el AMBA sólo el 76\% de la población posee agua potable por red y 57\% a cloacas (INDEC, 2010). Estos valores, a su vez, se modifican sustancialmente si se analiza de manera separada la cobertura de la CABA ( $97 \%$ en agua y $98 \%$ en cloaca) y los partidos del GBA (67\% y 41\%).

Tabla 1. Hogares con cobertura de agua potable y cloacas en el AMBA (2010). Fuente: elaboración propia en base a resultados del Censo Nacional de Población, Hogares y Viviendas INDEC (2010).

\begin{tabular}{|c|c|c|c|c|c|c|c|c|c|}
\hline \multirow{3}{*}{ Jurisdicción } & \multirow{3}{*}{ Total de hogares } & \multicolumn{4}{|c|}{ Agua de red } & \multicolumn{4}{|c|}{ Desagües cloacales } \\
\hline & & \multicolumn{2}{|c|}{ Hogares servidos } & \multicolumn{2}{|c|}{$\begin{array}{c}\text { Hogares no } \\
\text { servidos }\end{array}$} & \multicolumn{2}{|c|}{ Hogares servidos } & \multicolumn{2}{|c|}{$\begin{array}{c}\text { Hogares no } \\
\text { servidos }\end{array}$} \\
\hline & & Total & $\%$ & Total & $\%$ & Total & $\%$ & Total & $\%$ \\
\hline CABA & 1.150 .134 & 1.120 .818 & 97 & 29.316 & 3 & 1.128 .920 & 98 & 21.214 & 2 \\
\hline $\begin{array}{l}24 \text { partidos } \\
\text { GBA }\end{array}$ & 2.934 .373 & 1.967 .212 & 67 & 967.161 & 33 & 1.211 .000 & 41 & 1.723 .373 & 59 \\
\hline Total AMBA & 4.084 .507 & 3.088 .030 & 76 & 996.477 & 24 & 2.339 .920 & 57 & 1.744 .587 & 43 \\
\hline
\end{tabular}

La diferencia en los niveles de cobertura entre la CABA y el resto del AMBA se explica por el modo que asumió el proceso de producción urbana en la metrópolis desde fines del siglo XIX. Esto dio lugar, por un lado, a una Ciudad mayormente planificada y ordenada, con provisión de servicios básicos para casi toda su población (exceptuando las villas y asentamientos). $\mathrm{Y}$, por otro lado, a una gran periferia con un crecimiento desarrollado sin normas de regulación para la producción del suelo y sin la garantía de los servicios urbanos básicos, lo que llevó a sus pobladores a afrontar procesos de auto-urbanización y autoproducción de dichos servicios (Pírez, 2006). 
A esta dualidad centro-periferia y a la marcada diferenciación del eje norte-sur, se agregaron a partir de la década de 1990 nuevas formas de fragmentación urbana, como la aparición de barrios cerrados y countries en la periferia, que agravaron la segregación y los conflictos por el uso del territorio (Catenazzi y Reese, 2010). A ello se suman los procesos de privatización y descentralización que comenzaron en esa misma década y que han provocado un deterioro en el hábitat popular, incrementando la localización de asentamientos informales en zonas ambientales degradadas, es decir, en zonas inundables con suelos contaminados y carentes de servicios, muchas de ellas ubicadas en las principales cuencas metropolitanas (Merlinsky, 2016).

La falta de servicios de saneamiento en áreas ambientalmente degradadas incrementa el riesgo sanitario de los grupos sociales que residen en estos territorios. Ya sea por verse expuestos a la ingesta de agua de mala calidad o por afrontar inundaciones frecuentes con aguas contaminadas que hacen desbordar las aguas negras de los pozos ciegos, aumentan las probabilidades de contraer enfermedades hídricas (Tobías y Fernández, 2019).

En lo que refiere a las inundaciones en el AMBA, sus causas son diversas, pudiendo diferenciarse a grandes rasgos factores propios de las dinámicas climáticas y de los aspectos geofísicos del territorio, y factores propiamente antrópicos. Entre las causas climáticas se destacan principalmente el aumento progresivo de las precipitaciones - de $1000 \mathrm{~mm} / \mathrm{año}$ a $1200 \mathrm{~mm} / \mathrm{año}-$, su frecuencia e intensidad (tal como lo demostró el caso extremo en la ciudad de la Plata, fuera del AMBA, pero dentro de la mancha urbana de Buenos Aires, que en el año 2013 sufrió lluvias superiores a los $300 \mathrm{~mm}$ ). Así como también la mayor frecuencia e intensidad del fenómeno de la Sudestada, que actúa como tapón hidráulico impidiendo que las aguas del continente drenen hacia el río. ${ }^{1}$ Ambos factores se vieron incrementados en los últimos años producto del cambio climático (Barros y Camillioni, 2016) y su combinación aumenta las probabilidades de catástrofes en la región. También se destacan los factores geomorfológicos asociados a la baja permeabilidad de los suelos (producto en gran medida de factores antrópicos) y materiales superficiales, las bajas pendientes regionales, las planicies aluviales amplias y un alto nivel de la napa freática. ${ }^{2}$

Por su parte, las causas puramente antrópicas refieren a las consecuencias del proceso de urbanización de la región expresadas, en primer lugar, en el incremento de la pavimentación -y, por ende, en la impermeabilización de los suelos- y la remoción de la cubierta vegetal. En segundo lugar, en la cultura de la canalización y rectificación de los cursos de agua que primó en el seno de la ingeniería hidráulica durante los últimos siglos que, al no verse acompañada de obras de mantenimiento de los canales, llevó a su saturación, obstrucción y deterioro (González, 2018). En tercer lugar, en la ocupación de zonas anegables y en la acumulación de residuos en los cauces de las principales cuencas metropolitanas (Pereyra, 2014).

1 La Sudestada es un fenómeno meteorológico común en una extensa región del Río de la Plata caracterizado por el paso vientos fríos provenientes del sur hacia el cuadrante del sureste. Dado que la dirección del viento coincide con el eje del Río de la Plata, si este se prolonga varios días, su arrastre sobre las aguas del río interfiere con el normal desagüe del río, lo que produce el aumento del nivel del mismo sobre la costa argentina y dificulta el drenaje de cauces menores, provocando frecuentes inundaciones en áreas y municipios costeros.

2 La napa o capa freática es una acumulación de agua subterránea que se encuentra a una profundidad próxima al nivel del suelo. En las zonas más bajas del AMBA se encuentra a menos de $1 \mathrm{~m}$ de profundidad y es frecuente que aflore a la superficie, mientras que en las zonas altas se suele encontrar a 4 a $10 \mathrm{~m}$. La capacidad de almacenamiento de agua de los suelos en relación con las precipitaciones se encuentra condicionada a la profundidad de la napa, aumentando el escurrimiento superficial (y por ende la posibilidad de inundaciones) en forma directa con la disminución de la profundidad del nivel freático (Pereyra, 2014:263). 
GABRIELA MERLINSKY, MELINA TobíAS

En el AMBA, las zonas más afectadas por las inundaciones son las costeras susceptibles a la sudestada y las áreas bajo cota, generalmente próximas a las riberas de las principales cuencas metropolitanas, donde se asienta la población de menores recursos. En estos territorios las inundaciones traen consigo contaminación, ya que las aguas de los ríos metropolitanos presentan altos niveles de degradación ambiental, con presencia de metales pesados y efluentes orgánicos no tratados (Herrero y Fernández, 2008).

Como se observa en la Figura 2, las áreas más inundables coinciden con las tres cuencas metropolitanas que atraviesan la región: la CMR, la CRR y la cuenca del río Luján. Estos territorios comprenden gran parte de la población del área y la región metropolitana: mientras que en la CMR viven más de seis millones de habitantes, en la CRR habitan cuatro millones y en la del Luján casi tres millones.

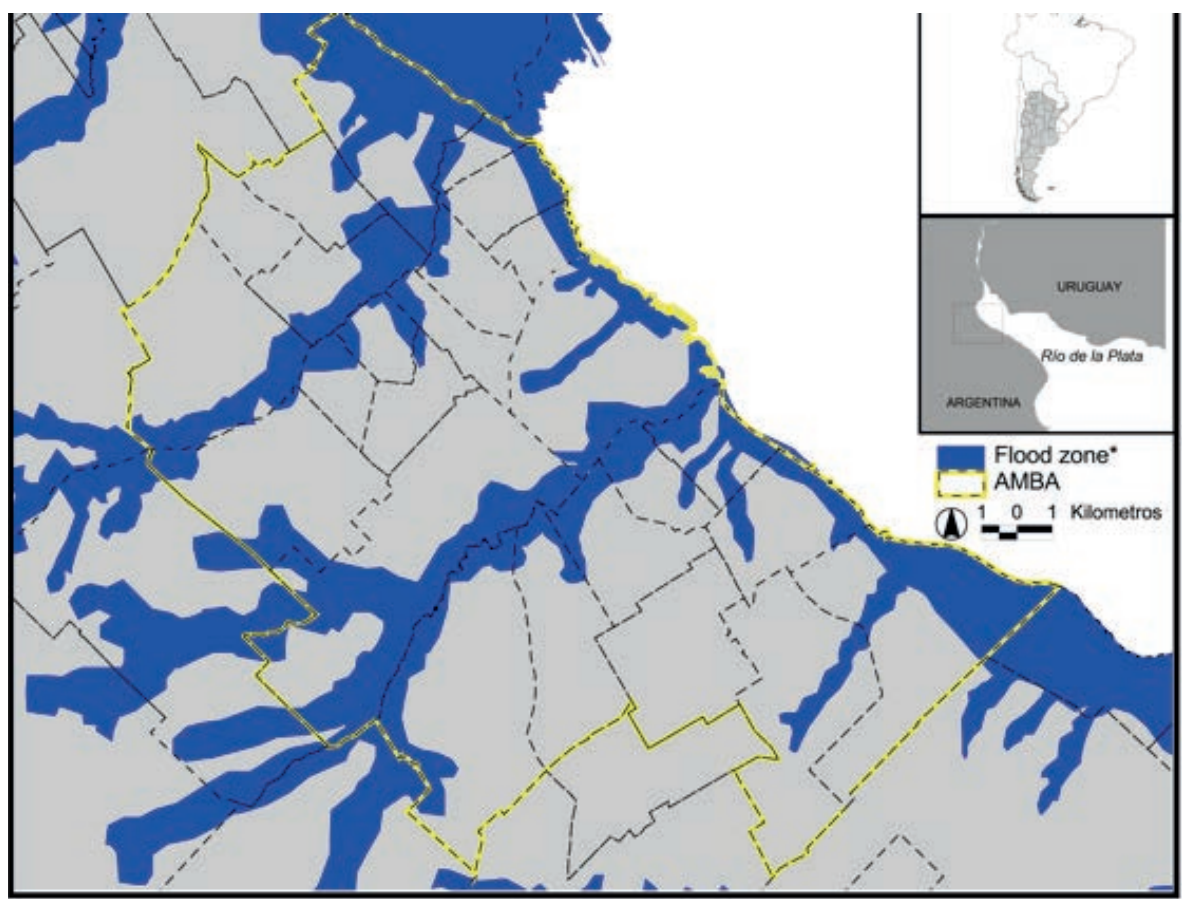

*Las zonas inundables han sido definidas en función de distintas unidades geomorfológicas (suelos con basamento cristalino, planicies aluviales y terrazas fluviales, delta, cordones litorales, delta y planicie de marea antigua).

Figura 2. Crecimiento urbano y territorios inundables del AMBA. Fuente: Elaboración propia en base a Nabel y Pereyra (2002).

La CMR y la CRR presentan altos niveles de vulnerabilidad socioambiental: población con NBI, contaminación de los suelos con metales pesados, basurales a cielo abierto, riesgo de inundación y falta de servicios básicos, especialmente en las márgenes de los ríos principales y arroyos tributarios (Defensor del Pueblo de la Nación, 2007; Merlinsky, 2016; Potocko, 2017). Una mirada multiescalar permite identificar los distintos colectivos y organizaciones sociales que reclaman por mejoras en las condiciones de vida en la ribera, exigiendo al Estado la implementación de políticas públicas que permitan superar las diferentes brechas en materia de justicia hídrica y ambiental. 


\section{La problematización pública de la cuestión hídrica en las cuencas de los ríos Matanza-Riachuelo y del río Reconquista}

Hemos visto que los territorios hidrosociales se moldean a través de prácticas materiales y significados embebidos en los procesos históricos de producción, apropiación y circulación del agua. Desde comienzos del presente milenio, las cuencas hídricas metropolitanas no solamente son escenarios de conflicto, sino que también han pasado a ser uno de los principales asuntos en disputa. Esto se debe a la relevancia política que ha adquirido la problematización pública de la cuestión ambiental en Argentina. El caso de la CMR es un claro ejemplo de esto. Su visibilidad pública comenzó en julio de 2004, cuando un grupo de vecinos y profesionales de la salud de la zona sur de la Cuenca Matanza-Riachuelo interpusieron una demanda judicial contra el Estado nacional, la provincia de Buenos Aires, el Gobierno de la CABA y 44 empresas, por daños y perjuicios sufridos a raíz de la contaminación del río Matanza-Riachuelo, solicitando asimismo la recomposición del ambiente dañado. Dos años después, la Corte Suprema de Justicia hizo lugar a la demanda en los aspectos vinculados con la prevención, recomposición y el resarcimiento del daño ambiental colectivo. En su resolución inicial determinó el objeto de la causa (la tutela del bien colectivo) e intimó a los gobiernos demandados a que presentaran un plan de saneamiento de la cuenca. Esto dio lugar a la creación de la Autoridad de Cuenca Matanza Riachuelo (ACUMAR), en tanto organismo interjurisdiccional encargado de la gestión de la cuenca (Merlinsky, 2013).

Sin embargo, la problematización de la cuestión hídrica adquiere significados muy diferentes si comparamos la CMR con la CRR, algo que tiene fuertes implicancias en materia de políticas públicas. En primer lugar, la problematización de la cuenca como cuestión central de política ambiental es diferente en cada caso: mientras la CMR representa un caso testigo donde en el último tiempo se ha logrado adoptar un enfoque socioambiental del territorio de cuenca, en la CRR este enfoque nunca llegó a configurarse como tal. Esto último se explica porque, en el primer caso, determinados actores con capacidad de influencia como la Defensoría del Pueblo de la Nación y diferentes ONG en defensa del ambiente han podido lograr incidencia en los tribunales (Merlinsky, 2013).

En segundo lugar, existen institucionalidades distintas encargadas de su gestión, que imprimen y llevan adelante diferentes miradas sobre la cuenca. Mientras que el proceso de judicialización que atravesó la CMR, y que dio lugar a la creación de la ACUMAR, implicó la adopción de un enfoque interjurisdiccional a través de la creación del Plan Integral de Saneamiento Ambiental que supera la mirada estrictamente ingenieril de las obras hidráulicas; en el caso de la CRR, la falta de un plan integral de recomposición y la histórica intervención de organismos internacionales de crédito asociados a la realización de obras de infraestructuras (reducción de inundaciones y la extensión de las redes de servicio de agua y cloaca) imprimió al Comité de Cuenca del Río Reconquista un enfoque sectorial muy restringido, con respuestas técnicas centradas exclusivamente en los problemas de la calidad y cantidad de agua.

Aquí es importante destacar que nuestra concepción sobre la gobernabilidad ambiental implica una comprensión de la autoridad como una categoría dinámica y relacional que requiere su renovación continua a través de compromisos entre el gobierno y los ciudadanos. La autoridad presupone legitimidad y reconocimiento y, como tal, implica esfuerzos concertados (por ejemplo, articulación, persuasión y negociación) para 
mantenerse (Bulkeley, 2012:2429). Como Nightingale (2018) sostiene, los gobiernos no tienen poder, pero en su lugar ejercen poder a través de instituciones, procedimientos y programas, y es el reconocimiento de ese poder lo que produce "estatalidad" entendida como la autoridad para gobernar.

En pocas palabras, la autoridad siempre está en formación y, por esa razón, es muy importante prestar atención al modo en que las diferentes disputas por el acceso al agua y por los modos de mitigar las inundaciones tienen lugar en estos territorios hidrosociales. En estos conflictos y controversias participan distintos actores: colectivos territoriales organizados, empresas prestadoras de servicio de agua potable y saneamiento, organismos municipales, provinciales e interjurisdiccionales (como los organismos de cuenca), empresas privadas (industrias, sector inmobiliario) y otros; que disputan las formas que asume la distribución y circulación del agua en la ciudad. En ese sentido, los conflictos también moldean la definición de la cuestión hídrica lo que genera importantes debates tanto en torno a la desigualdad socioespacial en la distribución del agua como en la construcción de definiciones de la justicia hídrica "desde abajo".

\section{"El agua no es confort, el agua es vida". Los conflictos por el agua en la CMR}

La CMR es una de las áreas ambientalmente más degradadas del AMBA. En cuanto a la problemática hídrica, los principales riesgos se asocian, primero, a la falta de accesibilidad a servicios de agua potable y de saneamiento. Según los datos publicados por la Autoridad de la Cuenca Matanza-Riachuelo (ACUMAR, 2021a, 2021b), el 21\% de la población de la cuenca carece de agua potable por red y más del $48 \%$ no tiene acceso a cloacas. Segundo, a la contaminación del curso principal de agua, sus tributarios y los suelos con metales pesados que afectan la calidad de vida de la población (principalmente el plomo). Y, tercero, la exposición a inundaciones en las zonas próximas a la ribera del río y a las áreas de baja cota, ya sea por crecida de los ríos, sudestadas, lluvias intensas o ascenso de los niveles freáticos (Bertoni, 2012). La cuenca baja, que coincide con la CABA y los partidos más consolidados del AMBA, presenta indicadores que, en algunas localidades, muestran altos niveles de vulnerabilidad sanitaria. En el partido de Lomas de Zamora, por ejemplo, sólo el 31\% de la población accede a redes de saneamiento (INDEC, 2010) y, si bien la cobertura de agua por red es elevada (98\%), sus valores bajan considerablemente en los barrios populares localizados en las márgenes del río Matanza-Riachuelo. En este partido, la falta de cloacas se superpone a la elevación de la napa freática, lo que hace aún más gravosas las consecuencias de las inundaciones (Merlinsky, 2017).

En este territorio surge en el año 2001 el Foro Hídrico de Lomas de Zamora, una organización nucleada a partir de las graves inundaciones que tuvieron lugar en ese período y que pusieron en evidencia la crítica situación ambiental en el municipio. En todos estos años de activismo, se fueron generando aprendizajes colectivos desde un enfoque centrado en la justicia ambiental que establece lazos entre las desigualdades ambientales, sanitarias y sociales. A su vez, se han construido redes y alianzas con otros actores (universidades, organizaciones políticas y socio-territoriales), lo que ha permitido generar una expertise propia sobre la temática hídrica que excede la escala local y se proyecta hacia una escala de cuenca (Tobías, 2018). Los reclamos fueron 
ampliándose con el tiempo, pasando de las obras hidráulicas para prevenir inundaciones a la necesidad de ampliar la cobertura de cloacas y de respetar el ordenamiento ambiental del territorio (principalmente la preservación de reservorios y humedales que permitan contener el agua en épocas de lluvias).

Un repertorio clave para visibilizar los reclamos es la realización de campañas de difusión para hacer exigible el derecho al agua y al ambiente sano, y lograr que se concrete en acciones efectivas y afirmativas de las políticas públicas. A partir del año $2012 \mathrm{el}$ Foro Hídrico junto a la Facultad de Ingeniería de la Universidad de Buenos Aires y a la Fundación Che Pibe encabezaron una campaña titulada "El agua no es confort, el agua es vida”, buscando mostrar diferentes caras de la desigualdad territorial en el acceso a los servicios básicos: mientras algunos vecinos poseen la red de agua de $\mathrm{AySA},{ }^{3}$ otros se abastecen a través de redes vecinales, perforaciones (sin control de calidad sobre las mismas) e incluso algunos reciben agua a través de camiones cisterna o conexiones clandestinas.

La falta de cloacas en las zonas de mayor riesgo sanitario no es una casualidad. No es que la gente no tenga cloacas porque no tiene ganas, sino que siempre son los pobres los que han sido relegados por el negocio inmobiliario, por las empresas (...) Sin embargo, a la larga, los costos de no hacer las obras públicas los está pagando el Estado a través de la salud (Entrevista a Alfredo, Foro Hídrico, 14 de abril de 2013).

El argumento de los integrantes del Foro Hídrico cuestiona este modelo de análisis de costo-beneficio, planteando que la satisfacción de las necesidades de la población que está en riesgo sanitario no puede ser postergada. En esta construcción de argumentos, el problema se inscribe públicamente como un reclamo al Estado por la discontinuidad en las obras de infraestructura y por la falta de criterios de justicia hídrica que puedan compensar las inequidades en el acceso a los recursos.

En años recientes, el Foro se ha concentrado en reclamos colectivos por el derecho a la salud. Aquí la herramienta más poderosa la constituyen los relevamientos comunitarios que buscan poner en evidencia una situación que definen como "emergencia sanitaria". Para ello, han trabajado con otras organizaciones y universidades en pos de realizar diferentes estudios epidemiológicos para el diagnóstico de la situación sociosanitaria en los barrios. El objetivo es producir datos que contribuyan a mostrar los nexos entre los problemas de contaminación del agua, la falta de servicios sanitarios y la situación de salud a escala local. Un punto clave es denunciar la ausencia del Estado en materia de estudios epidemiológicos, particularmente en lo que hace a los registros de historias clínicas que tomen en cuenta la relación entre los peligros ambientales y las afecciones a la salud.

Como hemos visto, si las disputas por la justicia hídrica implican debates sobre los recursos, el ejercicio de la autoridad y la exigibilidad de los derechos, lo que aquí podemos ver es que el acceso al recurso cloacal es un elemento gravitante en términos de acceso a la salud y que la existencia de una nueva autoridad de cuenca no garantiza que esto se concrete. En ese sentido, aun cuando la causa Mendoza es un caso testigo para

3 Agua y Saneamientos Argentinos (AySA) es la empresa dedicada a la prestación de agua corriente y cloacas para la CABA y el GBA. 
la política ambiental en Argentina, no garantiza que se cumpla un derecho humano fundamental: el derecho al agua. Esto último se debe a un tipo de intervención de los tribunales que está muy centrado en la garantía del derecho al ambiente sano de un modo abstracto (el saneamiento de la cuenca en general), pero que no toma en cuenta cómo se traduce esto en territorios en desventaja ambiental.

Lo cierto es que a más de una década de iniciada la causa judicial, la implementación de la política de recomposición ambiental ha ido generando efectos en términos de desplazamientos poblacionales y la construcción de planes de vivienda social. Este proceso ha ocurrido en el territorio ribereño que se delimita a 35 metros de distancia a ambas orillas del río y que (siguiendo una antigua denominación jurídica) es conocido como "camino de sirga". La orden judicial de "liberar la traza del camino de sirga" ha sido una de las más resistidas por la población residente, al tiempo que ha sido una de las medidas del Plan Integral de Saneamiento Ambiental que ha generado más impactos territoriales. Esta forma de reconceptualizar el problema por parte del organismo de cuenca implica dejar de lado, precisamente, un enfoque más integral de justicia ambiental y de justicia hídrica, ya que no alcanza con la relocalización de la población, sino que es necesario poder garantizar mejores condiciones ambientales, sociales y sanitarias.

\section{Las desigualdades que se esconden detrás de las infraestructuras. Los conflictos por el agua en los barrios del cauce viejo del río Reconquista}

En referencia a la CRR, es interesante la experiencia de los barrios localizados en el cauce viejo del municipio de San Fernando ubicado en la zona norte del AMBA, a 28km de distancia de la CABA. Sus límites son el río Luján y el Río de La Plata, y los partidos de Tigre y San Isidro, siendo uno de los municipios que integran la cuenca baja del río Reconquista. Según los datos del último Censo Nacional de Población (INDEC, 2010), en San Fernando residen 161.673 personas, con una densidad poblacional de 177hab/ $\mathrm{km}^{2}$. Allí, el 8,6\% de los hogares presentan NBI (4.247 hogares aproximadamente), tendencia que disminuyó al año 2001.

En la década de 1990 se realizaron obras de rectificación del río en la cuenca baja destinadas a aminorar el riesgo de inundación. Sin embargo, como advierte Ríos (2015), las técnicas hidráulicas implementadas y la dinámica de urbanización fueron conformando un escenario de riesgo de desastres desigual, producto de la lógica capitalista, que favoreció a algunas áreas y estratos sociales en detrimento de otras que han incrementado su exposición a los anegamientos.

Los barrios populares localizados próximos al cauce viejo del río se consolidaron a partir de los años setenta mediante diferentes procesos de autoconstrucción. Posteriormente, la trama urbana se modificó a partir de las obras de rectificación del río, situación que trajo aparejada importantes transformaciones a partir de programas y políticas de urbanización -como el Programa de Mejoramiento de Barrios (PROMEBA)- que, a partir de 2006, permitieron el acceso al territorio a servicios de agua, cloaca y pavimentación. Sin embargo, esto no implicó ni el fin ni la solución a los problemas hídricos que enfrentan los vecinos (Moreno, 2015). 
En este contexto, los habitantes del cauce viejo del río en San Fernando enfrentan cotidianamente problemas asociados a la falta de calidad y mantenimiento de las infraestructuras, como son los desbordes cloacales permanentes, la baja presión de agua corriente e, incluso, la aparición de nuevas zonas de anegamiento, producto de la elevación y pavimentación de determinadas áreas del barrio. Estos problemas se superponen con otras deficiencias en servicios urbanos básicos, como son la electricidad o la recolección de residuos, y especialmente con situaciones de vulnerabilidad en relación con la tenencia de la tierra (ya que, si bien se trata de barrios urbanizados, los habitantes siguen aguardando la regularización dominial de sus viviendas).

Desde hace más de veinte años se han desarrollado procesos de organización comunitaria, con el acompañamiento del Instituto Internacional de Medio Ambiente y Desarrollo-América Latina (IIED-AL), una ONG que ha realizado acciones de promoción en diversos barrios del partido para lograr la provisión de servicios descentralizados de agua y saneamiento. En ese marco, en los últimos años se ha podido avanzar en la realización del proyecto "Proyectando Nuestro Barrio" destinado a favorecer acciones que apunten a la producción social del hábitat a través de líneas de microcréditos. ${ }^{4}$ Dentro de esas acciones, se han llevado adelante, junto a otros actores como la Red de Promotoras por el Hábitat de San Fernando y el Programa Interdisciplinario de la Universidad de Buenos Aires sobre Cambio Climático (PIUBACC), distintos relevamientos y mapeos colectivos para identificar áreas de riesgo.

Los resultados de estos trabajos muestran que uno de los principales riesgos a los que hacen frente los habitantes se asocia a la calidad y el mantenimiento de las infraestructuras de servicios. En ese sentido, si bien los planes de mejoramiento de viviendas lograron consolidar el pavimento, la provisión de electricidad y las redes de servicio de agua y cloaca; la falta de mantenimiento de las redes y su inadecuada instalación lleva a problemas frecuentes de desbordes cloacales, falta de presión de agua, cortes de luz e incendios por la precariedad de la instalación eléctrica. A su vez, los mapas de riesgo muestran que las zonas más vulnerables a los problemas ambientales y sociales son aquellas linderas con el cauce viejo del río Reconquista, mientras que las viviendas próximas a la Autopista Panamericana tienen un menor nivel de exposición a los mismos. ${ }^{5}$

Si analizamos esta dinámica histórica desde la perspectiva de la justicia hídrica, lo que aquí se observa es que aun cuando se ha garantizado la accesibilidad al agua y al saneamiento, esto representa una condición necesaria, pero no suficiente. Para que tenga una verdadera repercusión en términos de salud comunitaria, la cobertura de servicios tiene que estar asociada a cambios profundos en la calidad de la infraestructura y en el mejoramiento de las viviendas. En términos de la institucionalidad del agua hay que destacar el rol prominente de una cultura de la canalización y rectificación de los cursos de agua, así como de las grandes obras de saneamiento auspiciadas por el paradigma de la ingeniería hidráulica. Este enfoque, presente históricamente tanto en los organismos de cuenca como en las empresas prestadoras de servicio de agua y saneamiento (Tobías, 2019), ha privilegiado el diseño y elaboración de obras de gran

4 Las acciones del IIED-AL pueden encontrarse en https://www.iied-al.org.ar/la-red

5 Los mapeos y relevamientos realizados por la Red se encuentran disponibles en el siguiente link: https:// www.youtube.com/channel/UCvLS-If8KoAPsvAZ3AS-rLw 
escala, que implican temporalidades de largo plazo y cuantiosos presupuestos. En tanto estas obras se concretan de forma parcial, es esta circunstancia, unida a la falta de inversión en políticas de hábitat para los sectores populares, la que genera una mayor exposición al riesgo sanitario y de inundación.

Finalmente, en lo concerniente a la exigibilidad de los derechos, la falta de atención pública a la problemática de la CRR, en comparación con lo que sucede en la CMR, es lo que genera menores oportunidades políticas para la existencia de campañas públicas (como hemos visto en el caso del Foro Hídrico).

\section{Reflexiones finales}

Hemos visto que los problemas de inequidad en el acceso al servicio de agua y saneamiento, así como la exposición a las inundaciones periódicas, son procesos gravitantes para la desigualdad socioambiental en Buenos Aires. La gestión hídrica depende de la capacidad de inversión de la empresa de agua y saneamiento que tradicionalmente ha tenido un modelo basado en la oferta. La red fluye desde el norte hacia el sur y desde las zonas altas hacia las bajas, con la lentitud propia de una infraestructura que estuvo pensada para abastecer a las zonas centrales y a la población con capacidad de pago. Para superar la brecha de desigualdad se requiere de un modelo de gestión que tome en cuenta las disparidades existentes en los territorios hidrosociales no solo en materia de agua, sino también, y fundamentalmente, en relación con el acceso a suelo urbano de calidad para los sectores populares. Ante un mercado al que no le interesa la demanda de los sectores populares y la insuficiencia de las políticas habitacionales, la única alternativa es la ocupación de suelo vacante, por lo general, en áreas inundables. Es por eso por lo que la desigualdad hídrica está asociada tanto a la falta de servicios (y/o su mala calidad) como así también al riesgo de inundaciones.

Si bien en los últimos años han ganado relevancia las políticas de gestión ambiental de las cuencas hídricas metropolitanas, una primera conclusión es que estas iniciativas no logran superar una gobernanza del agua fragmentada. El ejemplo de la CMR es muy elocuente porque, aun cuando la Corte Suprema de Justicia lo planteó como un caso testigo en términos de la exigibilidad del derecho al ambiente sano, la ejecución del Plan Integral de Saneamiento Ambiental de la CMR no logra incorporar aspectos estructurales que relacionen el agua y la salud. Y, como vimos con el ejemplo del Foro Hídrico, no se observa la existencia de esquemas de participación que tomen en cuenta la estrecha relación entre estos aspectos como eje de intervención en la cuenca.

El caso de la CRR muestra que una cultura de la canalización y rectificación de los cursos de agua auspiciada por la ingeniería hidráulica está enfrentada a una gestión ambiental que tome en cuenta los territorios hidrosociales, lo que supone, a su vez, reconocer la voz de los vecinos de la propia cuenca. En tanto las obras se concretan de forma parcial en los sitios más afluentes de la CABA, esto representa un ciclo de retiro del Estado en materia de usos del suelo y una intervención parcial mediante infraestructuras que no resisten el paso del tiempo.

Los conflictos aquí analizados permiten entrever que es necesario hacer lugar a otras maneras de concebir el agua, basadas en la exigencia del derecho a la salud y a un hábitat 
Conflictos por el agua en las cuencas..

GabRIELA MeRLINSKY, Melina Tobías

digno. Es importante reconocer la existencia de formas de manejo comunitario del agua y de procesos de toma de decisiones y de organización colectiva que producen otras formas de entender la justicia hídrica. Los colectivos movilizados, al reclamar por las afectaciones ambientales y los impactos en la salud, están planteando un problema de inequidad intergeneracional y abren, además, caminos para la discusión en torno a la apropiación de la renta urbana y sus efectos sobre la salud colectiva. 


\section{Q Bibliografía}

» ACUMAR (2021a). Población incorporada al área con servicio de red de agua potable. Recuperado de: https://www.acumar.gob.ar/indicadores/poblacionincorporada-al-area-servicio-red-agua-potable/

» ACUMAR (2021b). Población incorporada al área con servicio de red cloacal. Recuperado de: https://www.acumar.gob.ar/indicadores/poblacion-incorporadaal-area-servicio-red-cloacal/

» Barros, V. y Camillioni, I. (2016). La Argentina y el Cambio Climático. De la Física a la política. Buenos Aires: Eudeba.

»Bertoni, J.C. (2012). La problemática de las inundaciones urbanas, el caso de la Cuenca Matanza-Riachuelo. Voces en el Fénix, 3(20), 52-62.

» Boelens, R. y Zwarteveen, M. (2005). Anomalous water rights and the politics of normalization: collective water control and privatization policies in the Andean region. En D. Roth, R. Boelens y M. Zwarteveen (Eds.), Liquid Relations (pp. 97123). New Brunswick: Rutgers University Press.

» Boelens, R., Cremers, L. y Zwarteveen, M. (2011). Justicia Hídrica: acumulación de agua, conflictos y acción de la sociedad civil. Lima: Justicia Hídrica, IEP, Fondo Editorial PUCP.

» Boelens, R., Hoogesteger, J., Swyngedouw, E., Vos, J. y Wester, P. (2016). Hydrosocial territories: a political ecology perspective. Water International, 41(1), 1-14. DOI: https://doi.org/10.1080/02508060.2016.1134898

» Bulkeley, H. (2012). Governance and the geography of authority: modalities of authorisation and the transnational governing of climate change. Environment and Planning A: Economy and Space, 44(10), 2428-2444.

» Catenazzi, A. y Reese, E. (2010). Derecho a la Ciudad. Voces en el Fénix, 1, 72-75.

»Damonte, G. y Lynch, B. (2016). Cultura, política y ecología política del agua: una presentación. Anthropologica, 34(37), 5-12.

»Defensor del Pueblo de la Nación (2007). Informe especial cuenca del río Reconquista. Primera parte. Buenos Aires.

» Garay, D. y Fernández, L. (2013). Biodiversidad urbana. Apuntes para un sistema de áreas verdes en la región metropolitana de Buenos Aires. Buenos Aires: UNGS.

»González, S.G. (2018). Riesgo hídrico y planificación urbana en la ciudad de Buenos Aires. Estudios Del hábitat, 16(2), e047, 2-13.

»Herrero, A.C. y Fernández, L. (2008). De los ríos no me río. Diagnóstico y reflexiones de las Cuencas Metropolitanas de Buenos Aires. Buenos Aires: TEMAS.

» INDEC, Instituto Nacional de Estadística y Censos (2010). Censo Nacional de Población, Hogares y Vivienda 2010. Buenos Aires: INDEC.

» Merlinsky, M.G. (2013). Política y justicia ambiental en la metrópolis de Buenos Aires. El conflicto del Riachuelo. Buenos Aires: Fondo de Cultura Económica.

» Merlinsky, M.G. (2016). Mists of the Riachuelo. River Basins and Climate Change in Buenos Aires. Latin American Perspectives, 43(4), 43-55. 
» Merlinsky, M.G. (2017). Ecología política del agua y territorialización de las luchas sociales: la experiencia del foro hídrico de Lomas de Zamora. Anthropologica, 35(38), 119-143

» Moreno, L. (2015). Riesgo y políticas públicas: disputas en el proceso de urbanización de una 'villa de emergencia' en la cuenca del río Reconquista. En J. Viand y F. Briones (Comps.), Riesgos al Sur. Diversidad de riesgos de desastres en Argentina (pp. 21-34). Buenos Aires: Editorial Imago Mundi.

» Nabel, P. y Pereyra, F. (2002). El paisaje natural. Bajo las calles de Buenos Aires. Buenos Aires: Museo Argentino de Ciencias Naturales.

» Nightingale, A.J. (2018). The socioenvironmental state: political authority, subjects, and transformative socionatural change in an uncertain world. Environment and Planning E: Nature and Space, 1(4), 688-711.

" Orlove, B. y Caton, S. (2010). Water sustainability: Anthropological approaches and prospects. The Annual Review of Anthropology, 39, 401-415.

»Paerregaard, K. y Andersen, A.O. (2019). Moving beyond the commons/ commodity dichotomy: the socio-political complexity of Peru's water crisis. Water Alternatives, 12(2), 459-469.

»Pereyra, F. (2014). Inundaciones en el Área Metropolitana Bonaerense (AMBA) y Gran La Plata (GLP): causas, evaluación del riesgo y ordenamiento territorial. XI Simposio de la Asociación Internacional de Planificación Urbana y Ambiente (UPE 11). Buenos Aires: Facultad de Arquitectura y Urbanismo.

»Pereyra, A. (2014). El riesgo a las inundaciones en la trama de los eventos extremos. En C. Carballo y S. Goldberg (Dir.), Comunidad e información ambiental del riesgo. Las inundaciones y el río Luján (pp. 13-26). Buenos Aires: Editorial Dunken.

»Pírez, P. (2006). La privatización de la expansión metropolitana en Buenos Aires. Economía, Sociedad y Territorio, 6(21), 31-54.

»Potocko, A. (2017). La cuenca del río Reconquista en la planificación metropolitana de Buenos Aires (Argentina). Ámbito, problemas y propuestas. Urbe. Revista Brasileira de Gestão Urbana, 9(3), 443-455.

»Ríos, D. (2015). Ciudad, técnicas hidráulicas y riesgos de desastres por inundaciones. Las transformaciones recientes de la cuenca baja del río Reconquista. En J. Viand y F. Briones (Comps.), Riesgos al sur. Diversidad de Riesgos de desastres en Argentina (pp. 3-20). Buenos Aires: Editorial Imago Mundi.

»Solo, T., Gutman, P. y Dascal, G. (1990). Las aguas bajan turbias. Tecnologías alternativas para el saneamiento en el Gran Buenos Aires. Informes de Investigación $N^{\circ} 9$. Buenos Aires: Centro de Estudios Urbanos y Regionales (CEUR).

"Swyngedouw, E. (2004). Social power and the urbanization of water. Oxford: Oxford University Press.

》Swyngedouw, E. (2009). The Political Economy and Political Ecology of the HydroSocial Cycle. Journal of Contemporary Water Research \& Education, 142(1), 56-60.

» Swyngedouw, E. y Williams, J. (2016). From Spain's hydro-deadlock to the desalination fix. Water International, 41(1), 54-73.

» Tobías, M. (2018). Conflictos territoriales y controversias socio-técnicas por el 
Gabriela Merlinsky, Melina Tobías

acceso al agua en Buenos Aires. El caso del Foro Hídrico de Lomas de Zamora. Revista Pampa, 17, 81-106.

» Tobías, M. (2019). El desarrollo de redes de agua y saneamiento en Buenos Aires y la primacía del paradigma de la ingeniería heroica. En L. Menazzi y G. Jajamovich (Comps.), Saberes Urbanos (pp. 173-202). Buenos Aires: Teseo.

" Tobías, M. y Fernández, V. (2019). La circulación del agua en Buenos Aires: resonancias geográficas y desigualdades socioespaciales en el acceso al servicio. Cuadernos de Geografía: Revista Colombiana de Geografía, 28(2), 423-441.

\section{Gabriela Merlinsky / merlinsk@retina.ar}

Socióloga por la Universidad de Buenos Aires. Doctora en Ciencias Sociales (UBA) y Doctora en Geografía (Universidad París 8). Investigadora Principal del CONICET. Profesora Regular en UBA y Coordinadora del Grupo de Estudios Ambientales (GEA), Área de Estudios Urbanos, Instituto de Investigaciones Gino Germani (FSOC-UBA).

\section{Melina Tobías / melina.tobias@gmail.com}

Socióloga, Magister en Sociología Económica y Dra. en Ciencias Sociales y Geografía. Investigadora Asistente del Consejo Nacional de Investigaciones Científicas y Técnicas (CONICET) con sede en el Área Urbana del Instituto de Investigaciones Gino Germani (UBA). Profesora Adjunta de la carrera de Trabajo Social, Universidad Nacional de José C. Paz y Jefa de Trabajos Prácticos en la carrera de Sociología, UBA. 